\title{
Structural Analysis and Adsorbability onto the Corneal Epithelial Cells-Model Interface of Vitamin Nano-Emulsions
}

\author{
Maiko Matsuki ${ }^{*}$, Miyuki Miyake ${ }^{1}$, Taku Ogura ${ }^{1}$, Ryo Hyodo $^{1}$, Norio Tobori ${ }^{1}$, \\ Hideki Sakai ${ }^{2}$ and Otto Glatter ${ }^{3}$ \\ ${ }_{1}^{1}$ Functional Materials Science Research Laboratories, R \& D, LION CORPORATION (7-2-1, Hirai, Edogawa-ku, Tokyo 132-0035, JAPAN) \\ ${ }^{2}$ Faculty of Science and Technology, Tokyo University of Science (2641 Yamazaki, Noda, Chiba, 278-8510, Japan) \\ ${ }^{3}$ Department of Chemistry, University of Graz (Heinrichstrasse 28, Graz, 8042, Austria)
}

\begin{abstract}
O} / \mathrm{W}$ nano-emulsions can be used as effective drug carriers of hydrophobic active ingredients in an aqueous solution, because nano-emulsions are comparatively stable and their structure can be controlled by changing the compositions and the preparation methods. In this paper, we focused on vitamin $\mathrm{A}$ and its derivatives (VA), which are among the widely-used lipophilic active ingredients, and tried to develop the nano-emulsions, which can bring out the efficiency of VA for the healing of injured corneas, with the detailed structural analysis of them using the small-angle X-ray scattering (SAXS) method. As a result, we elucidated that the nano-emulsions bearing the hydrophobic oil/water interface can be prepared by decreasing the surfactant concentration against vitamins. Moreover, we clarified that the nano-emulsions composed of lower surfactant concentration tend to adsorb VA onto the corneal epithelial cells-model interface. Therefore it is necessary to prepare the nano-emulsions, which have the hydrophobic oil/water interface for improving the adsorbability onto cell membranes.
\end{abstract}

Key words: nano-emulsion, small-angle X-ray scattering, vitamin A, adsorbability, drug carrier

\section{INTRODUCTION}

Surfactants molecular assemblies such as micelles, vesicles, and emulsions, can solubilize or disperse various hydrophobic substances efficiently in water. Therefore, in the cosmetic, pharmaceutical and medical fields, they have attracted great attention as drug carriers ${ }^{1,2)}$, which can deliver the hydrophobic active ingredients into the aqueous environment.

Micelles can solubilize only a small amount of hydrophobic substances since the size of micelles is small, and the micelle structure is led to one state depending on the composition and the physicochemical condition, due to the equilibrium system.

On the other hand, emulsions can disperse a larger amount of hydrophobic substances in an aqueous solution than micelles. Moreover the emulsion structure can be controlled by changing compositions and preparation methods. It means that the functions of emulsions as drug carriers can be improved by controlling their structure. Especially nano-emulsions, with a size typically in the range of 20-200 nm, have high dispersion stability. The effective applications of them as various drug carriers have been strongly expected ${ }^{3,4)}$.

Vitamin A and its derivatives (VA), among the lipophilic vitamins, have cytodifferentiation and antioxidation effects and various physiological functions ${ }^{5}$, therefore it has been widely used in the cosmetic and medical fields and expected further applications as active ingredients. Then we considered that the effective nano-emulsions containing VA can be developed as drug carriers, which enable to make great use of the efficiency of VA, by controlling their structure.

In order to control the function of molecular assemblies in water by changing their structure, it is important to analyze their structure in detail and to evaluate the relationship between structure and function. The small-angle $\mathrm{X}$-ray scattering (SAXS) method ${ }^{6}$ is known as one of the powerful tools for structural analysis of assemblies in the solutions. Recently, many researchers have been taking advantage of the method, because various molecular assem-

\footnotetext{
*Correspondence to: Maiko Matsuki, Functional Materials Science Research Laboratories, R \& D, LION CORPORATION (7-2-1, Hirai, Edogawa-ku, Tokyo 132-0035, JAPAN)

E-mail: m-mai@lion.co.jp

Accepted June 9, 2014 (received for review April 11, 2014)

Journal of Oleo Science ISSN 1345-8957 print / ISSN 1347-3352 online

http://www.jstage.jst.go.jp/browse/jos/ http://mc.manusriptcentral.com/jjocs
} 
blies such as micelles and microemulsions can be analyzed.

Especially, a pair distance distribution function (PDDF: $P$ $(r)$ ), which is obtained by transforming a scattered inten$\operatorname{sity}(I(q))$ with the indirect Fourier transformation (IFT) ${ }^{7,8)}$ method or the generalized indirect Fourier transformation $(\text { GIFT })^{9-13)}$ method, and an electron density profile $(\rho(r))$, which is obtained by deconvolution ${ }^{14)}$ of the PDDF using DECON, enable to evaluate the detailed size, form and internal structure of the molecular assemblies.

For example, by using the PDDF and the electron density profile, M. Tomšič et al. reported the detailed analysis of the solubilized locus of alcohols in the micelles of polyoxyethylene (23) dodecyl ether ${ }^{15)}$. L. K. Shrestha et al. revealed the structural change of polyglycerol oleic acid ester reverse micelles in decane during the change of temperature and concentration of the surfactant and water ${ }^{16)}$.

To the best of our knowledge, there are many reports on structural analysis of micelles using the PDDF and the electron density profile as above. However there is no report on structural analysis of nano-emulsions.

In this paper, we report the structural details of the nano-emulsions containing VA analyzed with SAXS, and the relationship between the nano-emulsion structure and the effect of VA adsorbability onto the corneal epithelial cellsmodel interface.

\section{EXPERIMENTAL}

\subsection{Materials}

Polyoxyethylene (60) hydrogenated castor oil (HCO) was purchased from Nikko Chemicals Co., Ltd., retinol palmitate (vitamin A derivative: VA) was purchased from DSM Nutrition Japan Co., Ltd., $d l$ - $\alpha$-tocopherol acetate (vitamin E: VE) was purchased from Rikenvitamin Co., Ltd., and dipalmitoylphosphatidylcholine (DPPC), dipalmitoylphosphatidylethanolamine (DPPE) and sphingomyelin were purchased from NOF Co., Ltd., cholesterol was purchased from WAKO Co., Ltd. All materials were used without further purification.

\subsection{Methods}

2.2.1 Preparation of the nano-emulsions

We prepared the nano-emulsions with agent-in-oil method: In a light-shielding vial, HCO, VA and VE, which was used as an antioxidant ${ }^{17)}$, were mixed and stirred at $70^{\circ} \mathrm{C}$ to become homogeneous. Then the mixture was added to water, which was heated at $80^{\circ} \mathrm{C}$, and stirred at $600 \mathrm{rpm}$ for more than 10 minutes to disperse the mixtures sufficiently. All nano-emulsions were prepared with higher concentration of HCO than the critical micelle concentration (ca. $0.01 \mathrm{wt} \%)^{18)}$. It is notable that if emulsions were prepared with agent-in-water method, namely the mixture of VA and VE was added to the HCO solution, nano-sized emulsions were not obtained. Composition of the measured samples was listed in Table 1.

We measured the size of the nano-emulsions of the samples B, D, and E by Dynamic Light Scattering with cumulant method using DLS 8000 (Otsuka electronics Co., Ltd.). All nano-emulsions were monodisperse and the size of the nano-emulsions in sample B was $18.9 \pm 5.9 \mathrm{~nm}$, in sample D was $21.2 \pm 5.9 \mathrm{~nm}$, and in sample E was $31.2 \pm 2.2$ $\mathrm{nm}$. Their sizes were not so much changed and the creaming was not observed in all samples even after stored more than 1 year at room temperature.

2.2.2 Structural analysis of assemblies using SAXS

X-ray scattering data was recorded on a SAXSess camera (Anton Paar Co., Ltd.). The measuring system composed of a W3830-sealed glass bulb X-ray source (PANalytical Co., Ltd., Cu-Ka [wavelength $=0.154 \mathrm{~nm}]$ ), a multilayer film Goebel mirror, a block collimator, a semi-transparent beam stop, a vacuum-proof glass capillary cell, a TCS120 temperature controller, a vacuum housing, and an imaging plate detector.

The measurable range of scattering vector $(q)$ was $0.03<$ $q / \mathrm{nm}^{-1}<6$. Samples were enclosed in a vacuum-tight thin quartz capillary and measured at room temperature for 30 minutes. All data were normalized to the same incident primary beam intensity for the transmission calibration and were corrected for background scattering from the capillary and we used water for absolute intensity calibration ${ }^{19)}$. The analysis of scattering data was performed with an IFT software package.

2.2.3 Evaluation of zeta potential of the nano-emulsions

Zeta potential of the nano-emulsions was measured with the laser Doppler method using ELSZ-1000 (Otsuka electronics Co., Ltd.). All nano-emulsions were measured at $25^{\circ} \mathrm{C}$ without further dilution.

Table 1 Composition of the measured samples.

\begin{tabular}{|c|c|c|c|c|c|c|c|}
\hline \multicolumn{2}{|c|}{ Sample } & A & B & C & D & E & F \\
\hline nonionic surfactant & $\mathrm{HCO} / \mathrm{wt} \%$ & 1.0 & 1.0 & 0.75 & 0.50 & 0.25 & 0.10 \\
\hline active ingredient & $\mathrm{VA} / \mathrm{wt} \%$ & - & 0.028 & 0.028 & 0.028 & 0.028 & 0.028 \\
\hline antioxidant & $\mathrm{VE} / \mathrm{wt} \%$ & - & 0.050 & 0.050 & 0.050 & 0.050 & 0.050 \\
\hline \multicolumn{7}{|c|}{ water } & \multicolumn{7}{c|}{ balance } \\
\hline
\end{tabular}


2.2.4 Evaluation of VA adsorption onto the corneal epithelial cells-model interface.

The liposomes, used as biological-model membranes were prepared with the following procedure: The mixed lipids composed of $18.75 \mathrm{~mol} \%$ of DPPC, $18.75 \mathrm{~mol} \%$ of DPPE, $25 \mathrm{~mol} \%$ of sphingomyelin and $37.5 \mathrm{~mol} \%$ of cholesterol were dissolved in chloroform/methanol (50 vol./5 vol.) in a flask. The composition of mixed lipids was based on that of the human cornea ${ }^{20)}$. Then the solvent was dried under a stream of $\mathrm{N}_{2}$ and completely removed in vacuo providing a thin and homogeneous lipid film.

In order to obtain the liposome solution, water was added to the dried lipid film and incubated at $60^{\circ} \mathrm{C}$, and then dispersed with a vortex mixer. The size of the resulting liposomes was larger than $1 \mu \mathrm{m}$.

The adsorbed VA amount onto the liposomes was measured using the following method: First, $2 \mathrm{~mL}$ of liposome aq. $(5 \mathrm{mmol} / \mathrm{L})$ and $3 \mathrm{~mL}$ of each nano-emulsions, which is triple diluted with saline solution $(0.9 \mathrm{wt} \% \mathrm{NaCl})$ on the assumption of the usage of the nano-emulsions as eyedrops, were mixed. Note that, the size of the nano-emulsions did not so much changed just after the dilution. After stirring the mixed solution with a magnetic stirrer for $1 \mathrm{~h}$ at $35^{\circ} \mathrm{C}$, the liposomes and the nano-emulsions were separated through a syringe filter(MF-Millipore MCE Membrane 0.8 $\mu \mathrm{m}$, Merck Millipore Japan Co., Ltd.) by their size. The amount of VA in the filtrates and the measured samples was calculated by measuring the ultraviolet absorbance of VA, which exhibits a strong absorbance peak at $327 \mathrm{~nm}^{21}$, using the visible-ultraviolet spectrophotometer (MultiSpec-1500, SHIMADZU Co., Ltd.). By subtracting the VA amount in the filtrates from that in the measured samples, the adsorbed VA amount onto the liposomes was calculated. Note that a negligible amount of VA was adsorbed onto the filer.

\section{RESULTS AND DISCUSSION}

3.1 Structural analysis of molecular assemblies using SAXS/IFT method

3.1.1 Theory of the IFT method

For monodisperse globular particle systems having $n$ particles in unit volume, the experimentally obtained scattered intensity $I(q)$ can be described by the equation as follows;

$$
I(q)=\mathrm{n} P(q) S(q)
$$

Where $P(q)$ is the averaged form factor and $S(q)$ is the structure factor. These factors provide information on the internal structure of the particles and particle-particle interaction, respectively. The $P(q)$ is given by the Fourier transformation of the PDDF: $P(r)$ as

$$
P(q)=4 \pi \int_{0}^{\infty} P(r) \frac{\sin q r}{q r} d r
$$

The SAXS data for all measured samples were analyzed by the IFT method $(S(q)=1)$ due to the low concentration of all samples. The electron density profile $(\Delta \rho(r))$ was obtained by transforming the PDDF using deconvolution.

3.1.2 Evaluation of the HCO micelle and nano-emulsion structure.

In order to clarify the structural difference between $\mathrm{HCO}$ micelles and the nano-emulsions, we tried to evaluate their structural details using the PDDF and the electron density profile. The sample A and B, which were listed in Table 1 were measured.

The scattered intensity curves $(I(q))$ of the HCO micelles and of the nano-emulsions ${ }^{22)}$ were transformed into the PDDFs with the IFT method(Fig. 1). Both PDDFs show two peaks, which means both assemblies have a 'coreshell' structure. Note that the $r$ values at the $P(r)=0$ show the maximum diameter of the assemblies. The size of the HCO micelles was about $18 \mathrm{~nm}$, and the size of the nano-emulsions was larger $(\sim 21 \mathrm{~nm})$ than that of the HCO micelles. This implies that the vitamins are included in the HCO molecular assemblies.

Then, the electron density profiles were calculated by using the deconvolution method(Fig. 2). These profiles, represented by equidistant step functions show the distribution of the electron density difference to water from the central point of assemblies to the interface with bulk. When the electron density of bulk water was defined as a base $(\Delta \rho=0)$, the electron density of 'shell', which is composed of the hydrophilic moiety of surfactants in the assemblies and has a higher electron density than water, was described in positive y-axis area $(\Delta \rho>0)$. On the other hand, the electron density of 'core', which is composed of the hydrophobic moiety of surfactants in the assemblies

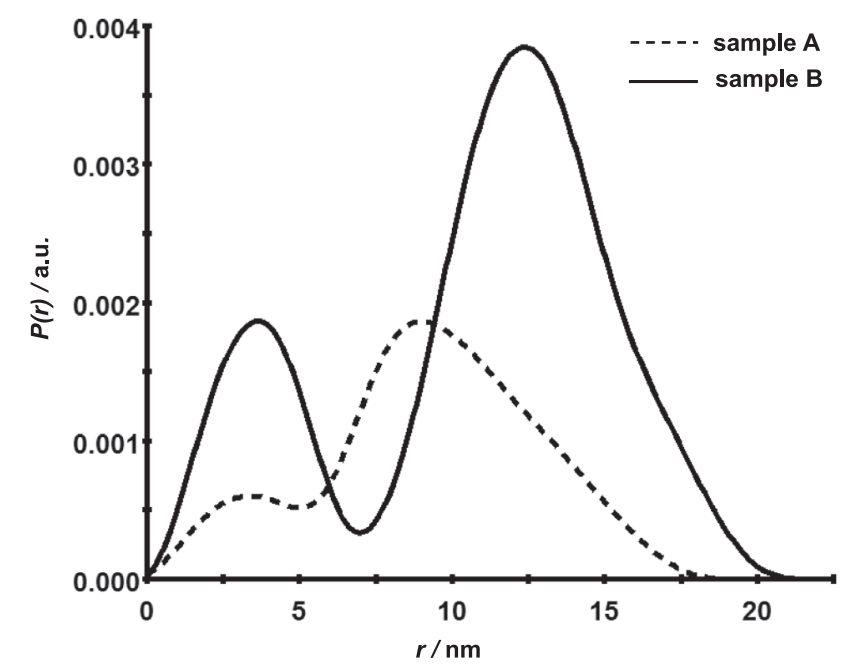

Fig. 1 The PDDFs of the HCO micelles (sample A) and of the nano-emulsion (sample B). 


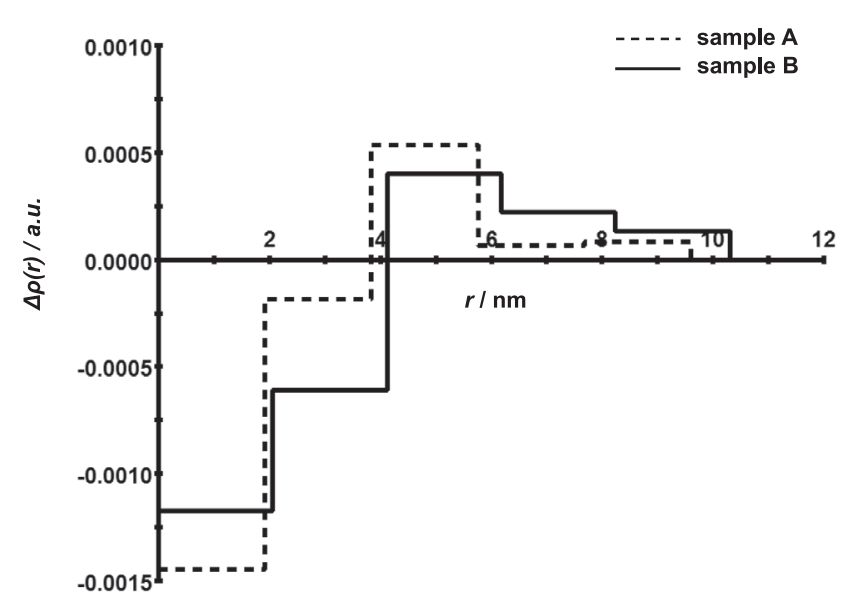

Fig. 2 The electron density profiles of the HCO micelles (sample A) and of the nano-emulsion (sample B).

and has a lower electron density than water, was described in negative $\mathrm{y}$-axis area $(\Delta \rho<0)$. The intersection point with $\Delta \rho=0$ describes the border between the core and the shell, and the $r$ value at that point describes the radius of the assemblies core.

When it comes to prepare O/W emulsions with a surfactant and a hydrophobic substance, the hydrophobic substance is contained into the emulsion core. In our system, the density profiles shown in Fig. 2 described the expansion of the core of the nano-emulsion compared with the HCO micelle, therefore the result suggests that the vitamins were contained into the core of the $\mathrm{HCO}$ assemblies.

3.1.3 Effect of the HCO concentration for the nano-emulsions.

As we mentioned above, the structure of the nano-emulsions would be controlled by changing the compositions and the preparation methods. Then we prepared various nano-emulsions composed of the same amount of vitamins and different amount of HCO in order to examine the structural changes of the nano-emulsions with different compositions. The nano-emulsions were prepared according to the procedure described in 2.2.1, and the sample B, $\mathrm{D}$, and $\mathrm{E}$ listed in Table 1 were measured.

The scattered intensity curves $(I(q))$ of the nano-emulsions $^{22)}$ were transformed into the PDDFs with the IFT method, and then the size and structure of the nano-emulsion droplets were evaluated. As shown in Fig. 3, all the PDDFs showed two peaks, which means all the nano-emulsions have the core-shell structure. With increasing of the HCO concentration, the size of the nano-emulsions decreased.

The electron density profiles were calculated from these PDDFs. Note that, the electron density of vitamins (VA: $307 / \mathrm{nm}^{3}$, VE: $\left.317 / \mathrm{nm}^{3}\right)$ were closer to that of water (334/ $\mathrm{nm}^{3}$ ) than that of alkyl chains of HCO (cf. triolein: $295 / \mathrm{nm}^{3}$ ). Therefore, on the hydrophobic site: 'core', the $\Delta \rho$ value

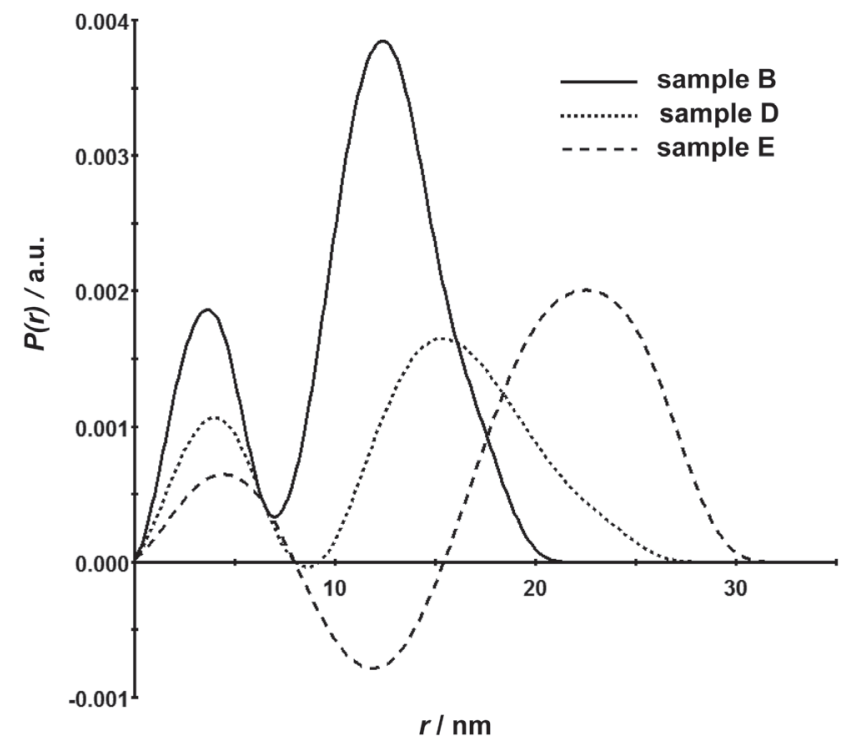

Fig. 3 The PDDFs of the nano-emulsions; The HCO concentration of sample B is $1.0 \mathrm{wt} \%$, sample D is $0.50 \mathrm{wt} \%$, and sample $\mathrm{E}$ is $0.25 \mathrm{wt} \%$ (listed in Table 1).

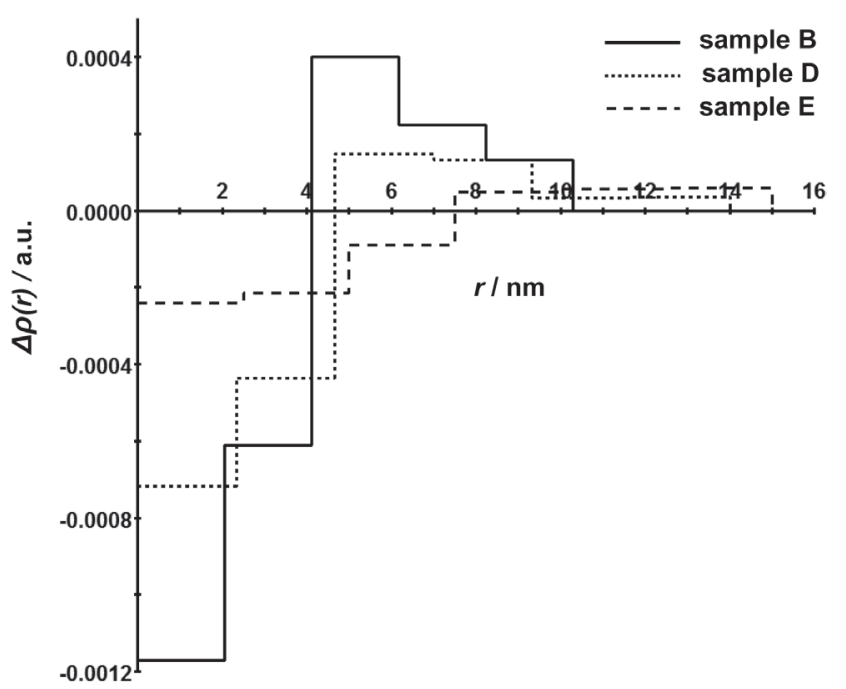

Fig. 4 The electron density profiles of the nano-emulsions; The HCO concentration of sample B is 1.0 $\mathrm{wt} \%$, sample $\mathrm{D}$ is $0.50 \mathrm{wt} \%$, and sample $\mathrm{E}$ is 0.25 $\mathrm{wt} \%$ (listed in Table 1).

tends to increase with increasing vitamins ratio in the nano-emulsions. On the other hand, on the hydrophilic site: 'shell', the $\Delta \rho$ value tends to decrease with decreasing amount of hydrophilic moiety of HCO, which covers the oil/ water interface of the emulsions.

Taking these facts into consideration, we interpreted the electron density profiles shown in Fig. 4 as follows. According to the profiles, the nano-emulsions composed of lower HCO concentration against VA have the smaller $\Delta \rho$ value of the core and also shell. The result suggests that the na- 


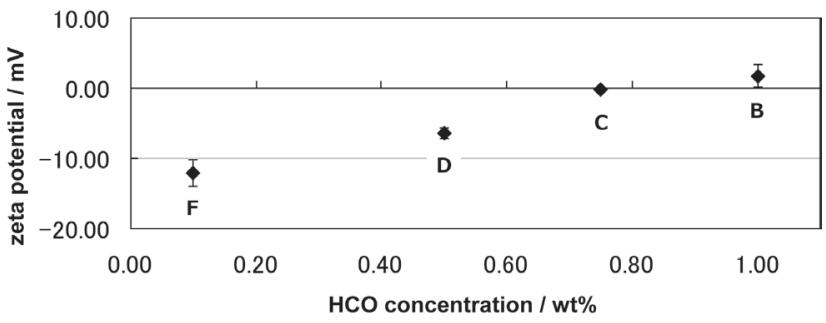

Fig. 5 The zeta potential of the nano-emulsions; The HCO concentration of sample B is $1.0 \mathrm{wt} \%$, sample $\mathrm{C}$ is $0.75 \mathrm{wt} \%$, sample $\mathrm{D}$ is $0.50 \mathrm{wt} \%$, and sample $\mathrm{F}$ is $0.10 \mathrm{wt} \%$ (listed in Table 1).

no-emulsions of lower HCO concentration are supposed to be covered with less HCO molecules per specific surface area of oil/water interface.

Therefore, we assumed that the nano-emulsions of lower HCO concentration have less hydrophilic, namely more hydrophobic oil/water interface than that of higher HCO concentration.

\subsection{Zeta potential of the nano-emulsions}

We tried to confirm the validity of our hypothesis: 'The nano-emulsions composed of lower HCO concentration have more hydrophobic oil/water interface than that of higher HCO concentration' in another way. Then, the zeta potential of the nano-emulsions was measured to examine the change of oil/water interface of the nano-emulsions. The sample B, C, D, and F listed in Table 1 were measured.

As the result shown in Fig. 5, the zeta potential of the nano-emulsions varied as the HCO concentration changed, and it tended to be more negative with decreasing the HCO concentration.

Hydrophobic substances bearing polar substituent groups are known to show the negative zeta potential ${ }^{23)}$, therefore in this case, vitamins are supposed to have the negative electric charge. Thus with increasing the HCO concentration, the negative charge derived from vitamins supposed to be shielded by the nonionic HCO molecules, which are not electrically charged, and came closer to 0 $\mathrm{mV}$ of the zeta potential ${ }^{24)}$.

The result also suggested that the nano-emulsions composed of lower HCO concentration supposed to be covered with less HCO molecules per specific surface area of oil/ water interface, and have more hydrophobic oil/water interface than that of higher HCO concentration.

\subsection{Evaluation of adsorbability of the nano-emulsions onto the corneal epithelial cells-model interface.}

As mentioned in 3.1.3 and 3.2, we confirmed that the nano-emulsions composed of the different HCO concentrations against VA have the different structures, namely the hydrophobicity of each emulsion oil/water interface is different. We assumed that this difference of the nano-emul-

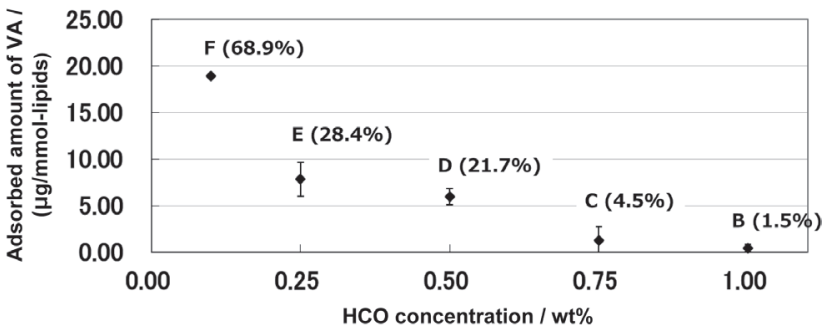

Fig. 6 Adsorbed amount (and proportion) of VA of the nano-emulsions onto the corneal epithelial cells-model interface; The HCO concentration of sample $\mathrm{B}$ is $1.0 \mathrm{wt} \%$, sample $\mathrm{C}$ is $0.75 \mathrm{wt} \%$, sample D is $0.50 \mathrm{wt} \%$, sample $\mathrm{E}$ is $0.25 \mathrm{wt} \%$, and sample $\mathrm{F}$ is $0.10 \mathrm{wt} \%$ (listed in Table 1).

sions interface would affect the adsorbability of VA onto the biological interfaces.

$\mathrm{VA}$ is known as an active ingredient for maintaining the health of eyes and healing of injured eyes by applying VA to the surface of eyes ${ }^{5,25,26)}$.

Therefore we examined the efficiency of the nano-emulsions as VA carriers to the corneal epithelial cell membranes using the liposomes, which were composed of the similar composition to lipids in the corneal epithelial cells. The preparation method was described in 2.2.4. Then we evaluated the adsorbability of VA according to the procedure described in 2.2.4. The sample B, C, D, E and F listed in Table 1 were measured.

The result was shown in Fig. 6. The vertical line describes the adsorbed amount of VA against per $1 \mathrm{mmol}$ of the mixed lipids, and the value in the parenthesis describes the proportion of the adsorbed amount of VA relative to its amount in the sample B, C, D, E, and F. According to the result, the nano-emulsions of lower HCO concentration, which are supposed to have comparatively hydrophobic oil/ water interface, tend to increase the VA adsorption ratio against the liposomes.

Generally, hydrophobic fat-soluble molecules are easy to interact with the lipid membranes, which are composed of hydrophobic layers. Therefore the nano-emulsions bearing more hydrophobic interface were supposed to interact effectively with the lipid membranes and increased the VA adsorbability.

Through the experiment, we considered the nano-emulsions, which have comparatively hydrophobic oil/water interface, would be effective carriers to deliver VA to the corneal epithelial cell membranes.

\section{CONCLUSION}

In conclusion, we demonstrated that the SAXS method, namely the PDDF and the electron density profile would be one of the powerful tools for the structural analysis of the 
nano-emulsion system. Through these analyses, the size and the internal structure of the nano-emulsions were elucidated. We also clarified that the nano-emulsions of lower HCO concentration were supposed to be covered with less HCO molecules per specific surface area of oil/water interface, and such the emulsions supposed to have more hydrophobic oil/water interface than that of higher HCO concentration.

Moreover, we successfully elucidated that the nanoemulsions of lower HCO concentration tend to increase the VA adsorption ratio onto the liposomes, which was used as the corneal epithelial cells-model interface. Therefore, the adsorption ratio of hydrophobic substances against lipid membranes can be increased by preparing the nano-emulsions, which have comparatively hydrophobic oil/water interface.

In our study, we demonstrated that nano-emulsions would be effective carriers of hydrophobic substances onto lipid membranes, especially onto the corneal epithelial cell membranes by controlling their structure.

\section{SUPPORTING INFORMATION}

The scattered intensity curves of the measured samples $\mathrm{A}, \mathrm{B}, \mathrm{D}$, and $\mathrm{E}$, and the PDDFs and the electron density profiles of the nano-emulsions prepared without an antioxidant VE were described in order to confirm the effect of $\mathrm{VE}$ on the nano-emulsion structure.

\section{References}

1) Janib, S. M.; Moses, A. S.; Mackay, J. A. Imaging and drug delivery using theranostic nanoparticles. Adv. Drug Deliv. Rev. 62, 1052-1063 (2010).

2) Rhee, Y.-S.; Mansour, H. M. Nanopharmaceuticals I: nanocarrier systems in drug delivery. Int. J. Nanotechnol. 8, 84-114(2011).

3) Solans, C.; Izquierdo, P.; Nolla, J.; Azemar, N.; GarciaCelma, M. J. Nano-emulsions. Current Opinion in Colloid \& Interface Science 10, 102-110 (2005).

4) Tadros, T.; Izquierdo, P.; Esquena, J.; Solans, C. Formation and stability of nano-emulsions. Adv. Colloid Interface Sci. 108-109, 303-318(2004).

5) Nollet, L. M. L. in Food Analysis by HPLC, Third Edition. (Nollet, L. M. L.; Toldra, F. ed.), CRC Press, pp. 271-324(2012).

6) Glatter, O. in Small-Angle X-Ray Scattering. (Glatter, O.; Kratky, O. ed.), Academic Press, London, pp. 119196 (1982).

7) Glatter, O. A new method for the evaluation of smallangle scattering data, J. Appl. Cryst. 10, 415-421 (1977).
8) Glatter, O. Determination of particle-size distribution functions from small-angle scattering data by means of the indirect transformation method. J. Appl. Cryst. 13, 7-11 (1980).

9) Brunner-Popela, J.; Glatter, O. Small-angle scattering of interacting particles. I. Basic principles of a global evaluation technique. J. Appl. Cryst. 30, 431-442 (1997).

10) Fritz, G.; Bergmann, A.; Glatter, O. Evaluation of small-angle scattering data of charged particles using the generalized indirect Fourier transformation technique. J. Chem. Phys. 113, 9733-9740(2000).

11) Bergmann, A.; Fritz, G.; Glatter, O. Solving the generalized indirect fourier transformation (GIFT) by Boltzmann simplex simulated annealing (BSSA). J. Appl. Cryst. 33, 1212-1216(2000).

12) Innerlohinger, J.; Wyss, H. M.; Glatter, O. Colloidal systems with attractive interactions: Evaluation of scattering data using the generalized indirect Fourier transformation method. J. Phys. Chem. B. 108, 18149-18157(2004).

13) Fritz, G; Glatter, O. Structure and interaction in dense colloidal systems: evaluation of scattering data by the generalized indirect Fourier transformation method. $J$. Phys. Condens. Matter 18, 2403-2419(2006).

14) Glatter, O.; Bernhard, H. Improvements in real-space deconvolution of small-angle scattering data. J. Appl. Cryst. 17, 435-441 (1984).

15) Tomšič, M.; Bester-Rogac, M.; Jamnik, A.; Kunz, W.; Touraud, D.; Bergmann, A.; Glatter, O. Ternary systems of nonionic surfactant Brij 35, water and various simple alcohols: Structural investigations by small-angle X-ray scattering and dynamic light scattering. $J$. Colloid Interface Sci. 294, 194-211 (2006).

16) Shrestha, L. K.; Dulle, M.; Glatter, O.; Aramaki, K. Structure of polyglycerol oleic acid ester nonionic surfactant reverse micelles in decane: Growth control by headgroup size. Langmuir 26, 7015-7024 (2010).

17) Because of low oxidation stability of VA, VE was added as an antioxidant. However, we confirmed that the nano-emulsions prepared without VE tends to show almost the same tendency as the nano-emulsions with VE. (See supporting information).

18) Saito, Y.; Sato, T. Micellar formation and micellar structure of poly (oxyethylene)-hydrogenated castor oil. Yakugaku zassi 112, 763-767 (1992).

19) Orthaber, D.; Bergmann, A.; Glatter, O. SAXS experiments on absolute scale with Kratky systems using water as a secondary standard. J. Appl. Cryst. 33, 218-225 (2000).

20) Broekhuyse R. M. The lipid composition of aging sclera and cornea. Ophthalmologica 171, 82-86(1975).

21) Cherng, S. H.; Xia, Q.; Blankenship, L. R.; Freeman, J. P.; Wamer, W. G.; Howard, P. C.; Fu, P. P. Photodecom- 
position of retinyl palmitate in ethanol by UVA lightformation of photodecomposition products, reactive oxygen species, and lipid peroxides. Chem. Res. Toxicol. 18, 129-138(2005).

22) The scattered intensity curves of the measured sample A, B, D, and E were listed in supporting information.

23) Kamogawa, K.; Akatsuka, H.; Matsumoto, M.; Yokoyama, S.; Sakai, T.; Sakai, H.; Abe. M. Surfactant-free O/ $\mathrm{W}$ emulsion formation of oleic acid and its esters with ultrasonic dispersion. Colloids Surf. A Physicochem. Eng. Aspects 180, 41-53(2001).
24) Barnes, T. J.; Prestidge, C. A. PEO-PPO-PEO block copolymers at the emulsion droplet-water interface. Langmuir 16, 4116-4121 (2000).

25) Toshida, H.; Odaka, A.; Koike, D.; Murakami, A. effect of retinol palmitate eye drops on experimental keratoconjunctival epithelial damage induced by $n$-heptanol in rabbit. Current Eye Research 33, 13-18 (2008).

26) Kim, E. C.; Choi, J.; Joo, C. A comparison of vitamin A and cyclosporine A $0.05 \%$ eye drops for treatment of dry eye syndrome. Am. J. Ophthalmol. 147, 206-213 (2009). 